\title{
Gravitationally bound geoengineering dust shade at the inner Lagrange point
}

\author{
R. Bewick, J.P. Sanchez, C.R. McInnes \\ Advanced Space Concepts Laboratory, University of Strathclyde, Glasgow, UK
}

\begin{abstract}
This paper presents a novel method of space-based geoengineering which uses the mass of a captured near Earth asteroid to gravitationally anchor a cloud of unprocessed dust in the vicinity of the $\mathrm{L}_{1}$ position to reduce the level of solar insolation at Earth. It has subsequently been shown that a cloud contained within the zero-velocity curve of the largest near Earth asteroid, Ganymed, can lead to an insolation reduction of $6.58 \%$ on Earth, which is significantly larger than the $1.7 \%$ required to offset a $2^{\circ} \mathrm{C}$ increase in mean global temperature. The masses of the next largest near Earth asteroids are found to be too small to achieve the required level of insolation reduction, however, they are significant enough to be used as part of a portfolio of geoengineering schemes.
\end{abstract}

Keywords: geoengineering Lagrange point four-body problem zero-velocity curve

\section{Introduction}

The current consensus is that climate change is not only happening but is almost unavoidable. Projections made using climate models over recent years have suggested that the mean global temperature is likely to increase by $1.1-6.4^{\circ} \mathrm{C}$ by the end of this century [6]. With the continuing industrialisation of the developing world and the lack of an agreed international protocol on tackling greenhouse gas emissions, this temperature increase seems unstoppable. While the focus of international efforts should remain with attempts to prevent climate change by the reduction of greenhouse gas emissions, it is prudent to investigate methods to mitigate its effects. This can be achieved by the deliberate manipulation of the Earth's climate, commonly referred to as climate engineering or geoengineering.

Several methods of space-based geoengineering have been proposed that have the goal of directly reducing the flux of solar radiation reaching the Earth's atmosphere. It has been predicted that a 1.7\% reduction in solar insolation will offset the effects of a global increase in temperature of $2^{\circ} \mathrm{C}$ [5]. These space-based schemes involve either placing solid reflective or refractive disks along the Sun-Earth line or dispersing large clouds of dust $[1,7,9,14,2]$. The methods that propose using reflective or refractive disks, such as that proposed by Angel [1], have the major disadvantage of requiring highly engineered devices manufactured either in-situ or terrestrially and launched into position. Due to their simplicity, dust cloud schemes do not have this disadvantage but typically require a much greater total system mass owing to the dispersion of the dust particles due the combination of the effects of gravity and solar radiation pressure. This work will investigate the novel possibility of using a captured near Earth asteroid, see [10], positioned at the first Lagrange point as both an in-situ source of material and to gravitationally 'anchor' a dust cloud at this point to greatly reduce the dust particle dispersion. An impression of this method can be seen in Fig. 1. This anchoring scheme will be achieved by placing a captured near Earth asteroid at the Earth-Sun $\mathrm{L}_{1}$ point therefore creating an artificial four-body problem. The size of cloud that can be anchored at the $\mathrm{L}_{1}$ point will then be estimated and the potential for solar radiation management will be determined by the use of a solar radiation model as described

*russell.bewick@strath.ac.uk 
in other work by the authors [2]. It will be assumed that the near Earth asteroid is captured using a continuous thrust methodology such as a mass driver [8] which can both liberate dust and maintain the asteroid close to the naturally unstable $\mathrm{L}_{1}$ point.

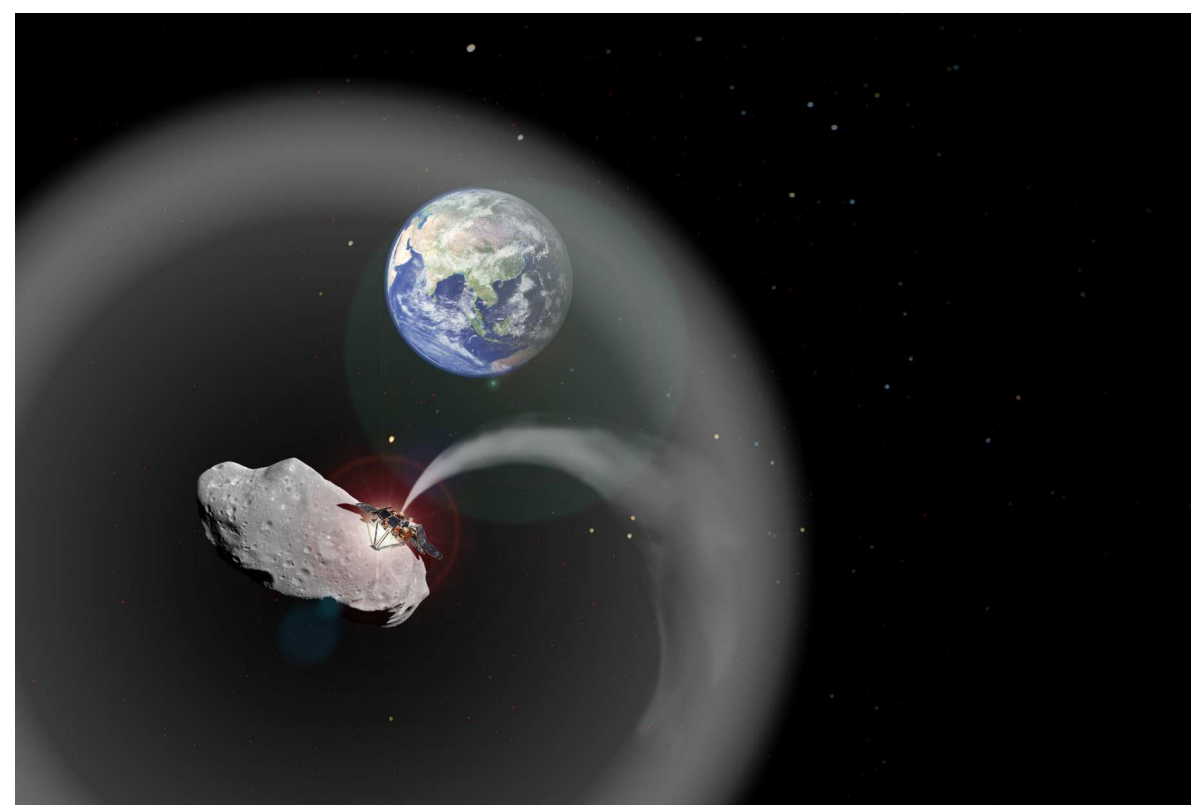

Figure 1: Impression of an $\mathrm{L}_{1}$ positioned dust cloud for space-based geoengineering.

\section{Four Body Problem}

The dimensionless equations of motion of a dust grain in the circular restricted Sun-Earth three-body problem (CR3BP), where the dust grain is considered to be the third body, in a rotating reference frame are given by [11];

$$
\begin{aligned}
\ddot{x}-2 \dot{y} & =\frac{\partial U}{\partial x} \\
\ddot{y}+2 \dot{x} & =\frac{\partial U}{\partial y} \\
\ddot{z} & =\frac{\partial U}{\partial z}
\end{aligned}
$$

where the distances are shown in Fig. 2 where $U$ is the effective three-body potential. These equations can be modified to define a circular restricted four-body problem (CR4BP) which includes a small asteroid captured at $\mathrm{L}_{1}$, where the new effective potential is defined by;

$$
U=\frac{1}{2}\left(x^{2}+y^{2}\right)+\frac{1-\mu}{\rho_{1}}+\frac{\mu}{\rho_{2}}+\frac{\gamma}{\rho_{3}}
$$

The parameter $\gamma$ is the mass fraction of the asteroid in relation to the mass of the three-body system, $\gamma=$ $m_{A} /\left(m_{s}+m_{E}\right)$, and $\mu$ is the mass ratio of the Earth to the mass of the Earth and Sun, $\gamma=m_{E} /\left(m_{s}+m_{E}\right)$. The scalar distances $r_{1}, r_{2}$ and $r_{3}$ are the separations between the primary and secondary bodies, the asteroid and dust particle respectively, as shown in Fig. 2.

Due to the small dust grain sizes involved in this study the effect of solar radiation pressure, quantified by the lightness parameter $\beta$, must be considered. It has been shown in [2] that above a grain radius of 


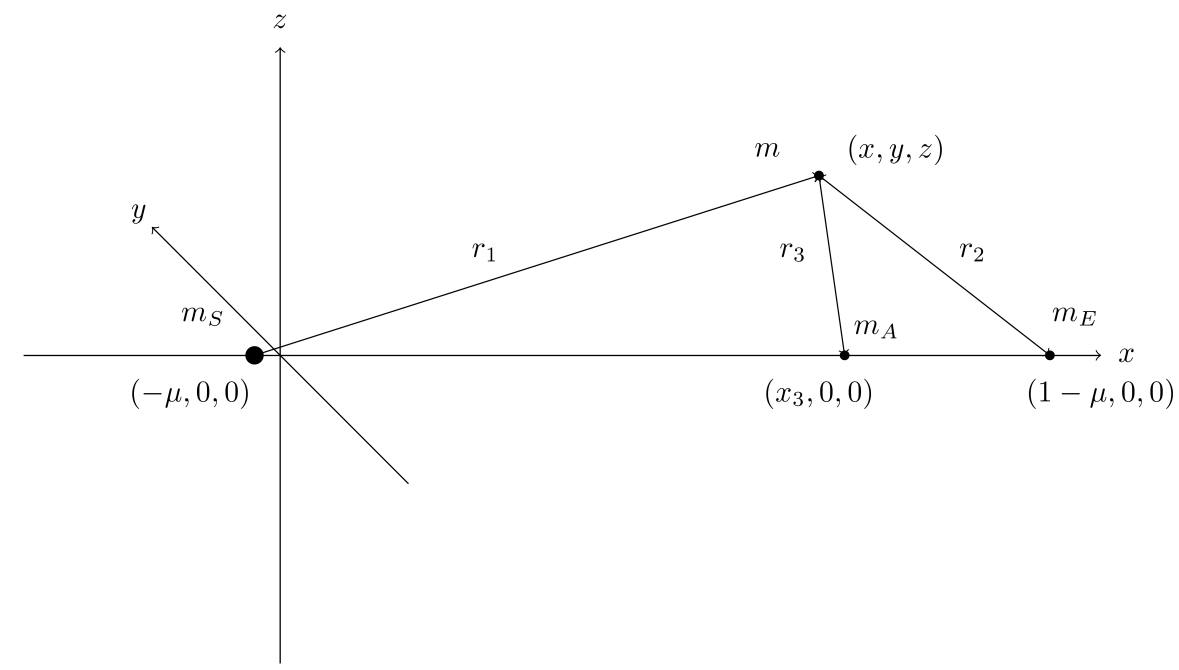

Figure 2: Four-body problem with Sun $m_{S}$, Earth $m_{E}$, asteroid $m_{A}$ and dust particle $m$.

$0.1 \mu \mathrm{m}$ solar radiation pressure is the dominant preturbation. The next strongest perturbation, the solar wind in a state of coronal mass ejection, is two orders of magnitude smaller. The parameter $\beta$ is the ratio of solar radiation pressure force to solar gravitational force experienced by the dust particle. This is described fully in [2] and its effect can be summarized as reducing the effect of solar gravitation by $(1-\beta)$ which results in the classical $\mathrm{L}_{1}$ Lagrange point equilibrium position along the Earth-Sun line being shifted Sunwards. For example a value of $\beta=0.005$ results in a shift of approximately $2,500 \mathrm{~km}$ whilst a value of $\beta=0.061$ would lead to a shift of $32,000 \mathrm{~km}$. Thus any object with a non-zero value of $\beta$ placed at the conventional $\mathrm{L}_{1}$ point would have a shorter instability timescale. When the gravitational potential of a body placed at the classical $\mathrm{L}_{1}$ point is considered, two new equilibrium positions appear. From the gradient of the potential $U$ defined in Eq. 2, the location of these new equilibria is defined by the position vector:

$$
x: x-\frac{1-\mu}{(x+\mu)^{2}}+\frac{\mu}{(x+\mu-1)^{2}} \pm \frac{\gamma}{\left(x-x_{3}\right)^{2}}=0
$$

and are located on the Sun-Earth line on either side of the classical $\mathrm{L}_{1}$ position, as shown in Fig. 3 and Fig. 4. These new equilibria, like the conventional $\mathrm{L}_{1}$ position, are unstable, but bound the asteroid, thus approximating the size of the dust cloud.

\section{Zero Velocity Curve}

The speed of a particle in the artificial 4-body system can be described by the Jacobi integral as;

$$
V^{2}=2 U(x, y, z)-C
$$

where $V$ is the particle speed and $C$ is the Jacobi constant. Since kinetic energy can only be strictly positive, it follows from Eq. 4 that the particle can only move within a region delimited by a zero velocity curve (i.e., when the right hand side of Eq. 4 vanishes). This constraint can be used to investigate the size of the region around the third body, the asteroid at $\mathrm{L}_{1}$, where a particle can become trapped if the energy, or Jacobi constant, of the particle is not large enough for escape. It is assumed that within this region particles are assumed to be collisionless, as will be confirmed later. Clearly, the maximum enclosed volume will be found for a zero velocity surface with a Jacobi constant equal to that of one of the new equilibrium points in the CR4BP. Combining Eq. 2 and Eq. 4 and noting that the equilibrium 
points lie on the $x$-axis the Jacobi constant for these positions can be found using;

$$
C=x^{2}+y^{2}+2\left(\frac{1-\mu}{|x+\mu|}+\frac{\mu}{|x+\mu-1|}+\frac{\gamma}{\left|x-x_{3}\right|}\right)
$$

where $x_{3}$ is the position of the asteroid, again shown in Fig. 2.

By numerically solving Eq. 5 to find the positions of the new equilibrium points for a body of a given mass these two values of $C$ can be then found. The surfaces that arise from this analysis can then be found and the shape and volume fully enclosed by the surfaces can be determined. Examples of these surfaces can be seen in Fig. 3 and Fig. 4 for an asteroid with a mass of $1 \times 10^{15} \mathrm{~kg}$ placed at the classical $\mathrm{L}_{1}$ position for $\beta=0$ and for $\beta=0.001$ respectively. It can be seen that for even small values of $\beta$ the shape of the zero-velocity curve becomes distorted and shrinks in size. The volume and approximate width of the zero velocity surfaces for a selection of near Earth asteroids, discussed later, can be seen in Fig. 5. The lack of results for the $\mathrm{L}_{1}$ case in Fig. 5 arises because the contour with the Jacobi constant of the equilibrium positions no longer bounds the asteroid position. Zero velocity curves still exist for these asteroids though they are greatly reduced in size. As expected both the width of volume enclosed is higher for an asteroid placed at the new displaced equilibrium position. Hence it can be concluded that an asteroid should be positioned at the slightly displaced equilibria to gain the maximum reduction in solar insolation.

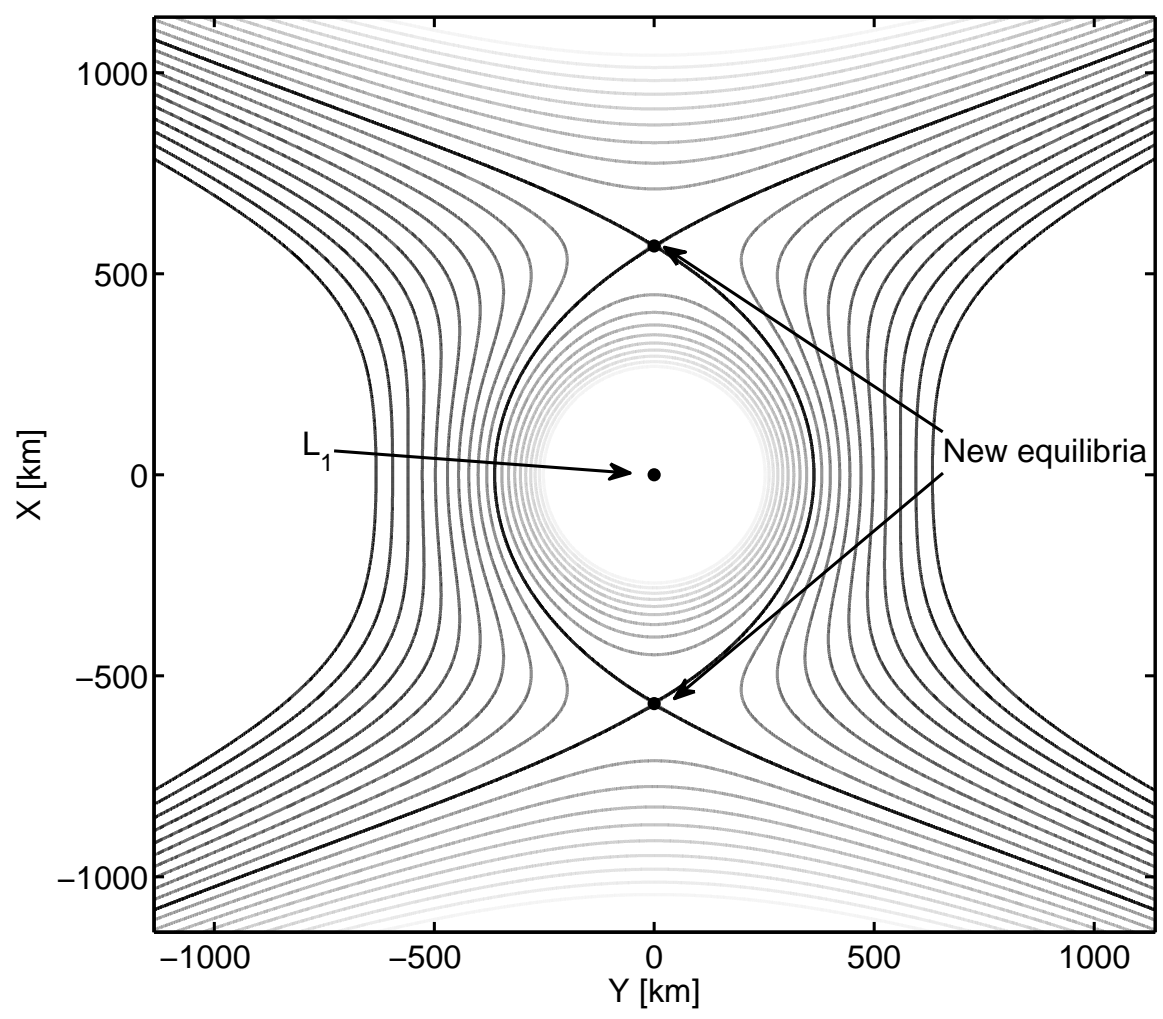

Figure 3: Contour plot showing the variation in the effective potential of the four-body problem for a body of mass $1 \times 10^{15} \mathrm{~kg}$ placed at the conventional $\mathrm{L}_{1}$ point for $\beta=0$, with bold lines showing the contours with the Jacobi constant of the equilibrium points. 


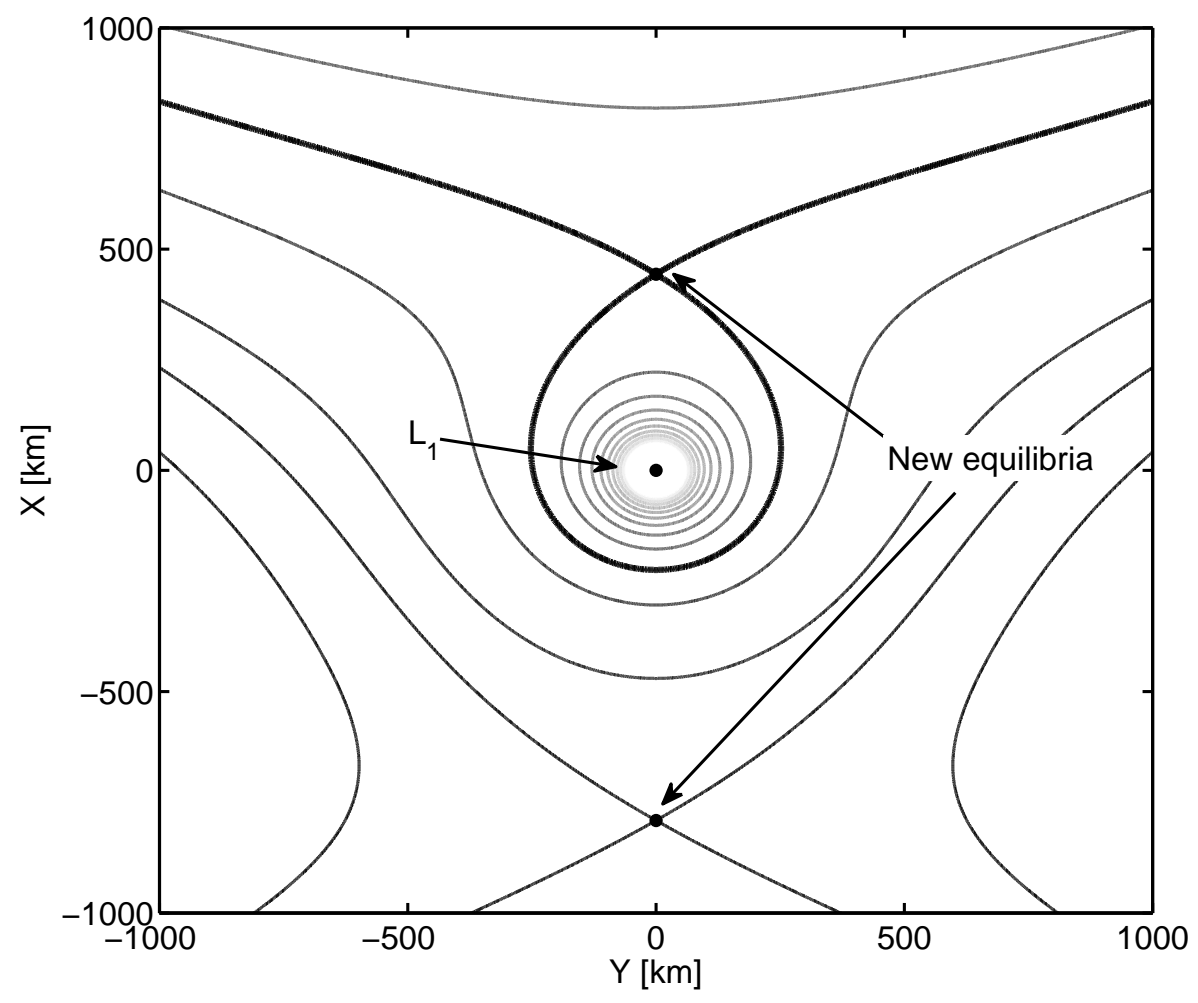

Figure 4: Contour plot showing the variation in the effective potential of the four-body problem for a body of mass $1 \times 10^{15} \mathrm{~kg}$ placed at the conventional $\mathrm{L}_{1}$ point for $\beta=0.001$, with the bold line showing the contour with the Jacobi constant of the equilibrium point that encloses the asteroid.

\section{Effect on Solar Insolation}

The effect that the dust cloud, confined by the zero velocity surface, has on the solar insolation has been determined for a set of real asteroids. Firstly, in order to assess the best set of candidates for the geo-engineering scheme proposed, a complete list of near Earth objects was retrieved from the NASA NEO program database ${ }^{1}$. Even if only the absolute magnitude of each object is known, the approximate mass of each near Earth asteroid can be estimated by means of Bowell et al.'s relation [3] and assuming an average density and albedo (i.e. $2,600 \mathrm{~kg} \mathrm{~m}^{3}$ and 0.154 [4]). Then the minimum $\Delta v$ of the Lambertarc connecting the asteroid and the Earth is optimized for the 250 largest objects, which includes all objects larger than $1 \times 10^{13} \mathrm{~kg}$. Next, a Pareto front with the largest objects and lowest transfer impulse (i.e. $I=m_{A} \dot{\Delta} v$ as a measure of incremental engineering effort) can be constructed leaving a set of 28 candidates as gravitational anchors. The Pareto front is shown in Fig. 6 and provides the list of the, a priori, most efficient asteroids to capture with masses ranging from $1 \times 10^{13} \mathrm{~kg}$ to the largest known near Earth asteroid mass of approximately $1.3 \times 10^{17} \mathrm{~kg}$ for the asteroid 1036 Ganymed. The impulse obtained from the Lambert arc method is used here as a sorting parameter only, since we envisage continuous low thrust used for capture.

The masses of these near Earth asteroids range from $1 \times 10^{13}-1.3 \times 10^{17} \mathrm{~kg}$. It was previously stated that the optimum position is likely to be the displaced equilibrium position, however, it must be determined whether it is feasible for asteroids of such large mass to be so displaced. The acceleration required to maintain the position can be determined using Eq. 1 by assuming $\dot{y}=\dot{x}=0$. This gives a result of $9 \times 10^{-7} \mathrm{~ms}^{-2}$, which is small, as would be expected. However, when the force required

\footnotetext{
${ }^{1}$ Data available online at http://neo.jpl.nasa.gov/ [retrieved 11/2010].
} 


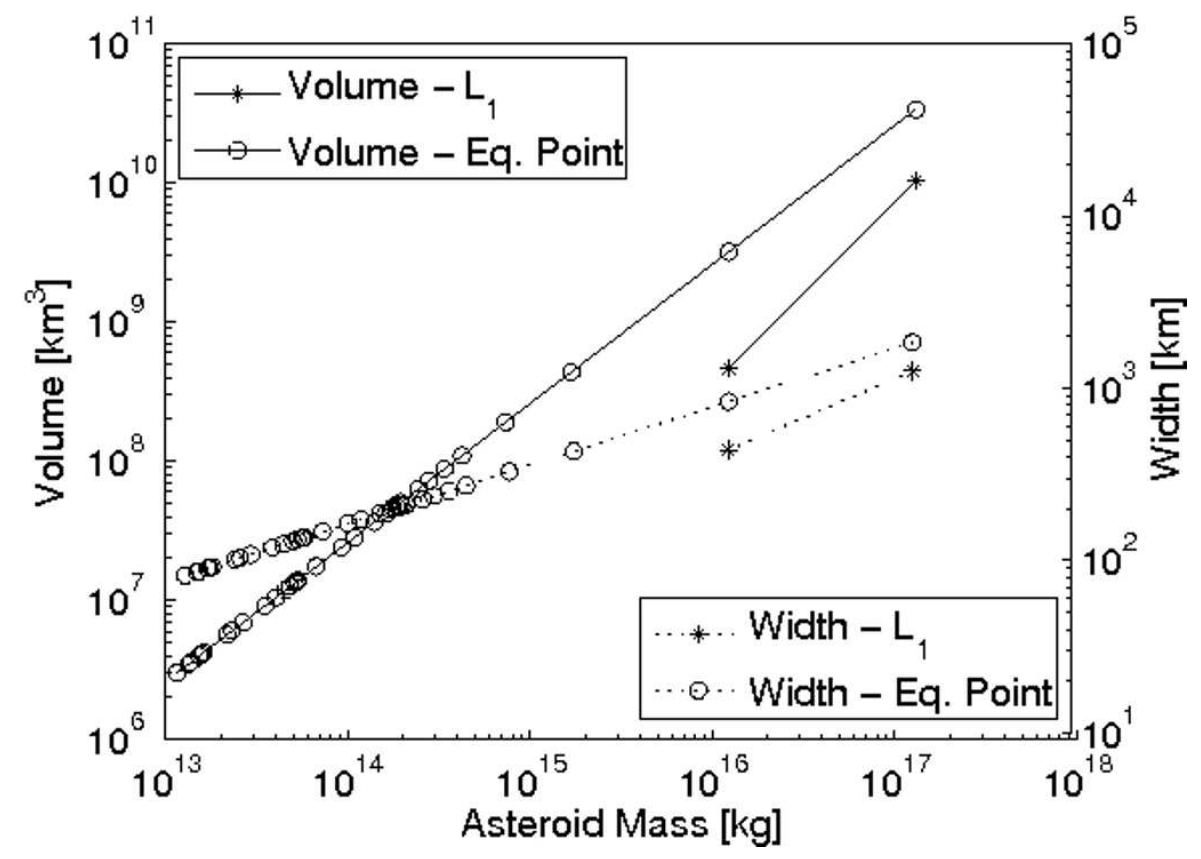

Figure 5: The width of the zero velocity curve and the volume it encloses for a selection of asteroid masses placed at the conventional $\mathrm{L}_{1}$ and new displaced equilibrium positions assuming grains with a value for $\beta$ of 0.005 .

to maintain the position is calculated the displaced equilibrium point no longer appears feasible. The insolation change for both positions shall be calculated as a comparison.

The maximum possible change in solar insolation was calculated for the masses on the Pareto front for both positions for a dust grain size of $32 \mu \mathrm{m}$, equivalent to a $\beta$ value of 0.005 [15]. This grain size was chosen as representative of real material.

The insolation reduction was computed using a numerical solar radiation model developed by the authors, whose details can be found in [2]. Specifically relating to this study, where the maximum acheivable insolation reduction is desired, the model initially assumes that all light passing through the zero velocity curve is blocked. These results, Fig. 7, show a linear trend on a log-log plot with the maximum insolation reduction of $6.58 \%$ being achieved for the asteroid Ganymed at the displaced equilibrium position, as was expected, with a maximum insolation reduction of $3.3 \%$ for the $\mathrm{L}_{1}$ position. This result meets the required $1.7 \%$ reduction in solar insolation required to offset expected climate change. To acheive an insolation change of $1.7 \%$ a homogeneous number density within the zero velocity curve, at the $\mathrm{L}_{1}$ position, of $120 \mathrm{~m}^{-3}$ is required, This gives a mean free path of approximately $10,000 \mathrm{~km}$. The initial velocity from the surface of Ganymed, assuming a radius of $31.7 \mathrm{~km}$, can be calculated using Eq. 4 to be $23.3 \mathrm{~ms}^{-1}$. This results in a collision timescale of 5.3 days, thus validating the assumption of collisionless particles. The maximum change in solar insolation reduces significantly for the next largest asteroid, to $1.42 \%$ and $0.42 \%$ for the displaced equilibrium and $\mathrm{L}_{1}$ positions respectively. These values not meet the required $1.7 \%$ insolation reduction noted previously to offset anticipated global warming. However, these results and some of the insolation reductions achieved by the next few asteroids could still be significant enough to be considered as part of a portfolio of geoengineering schemes to offset global warming. 


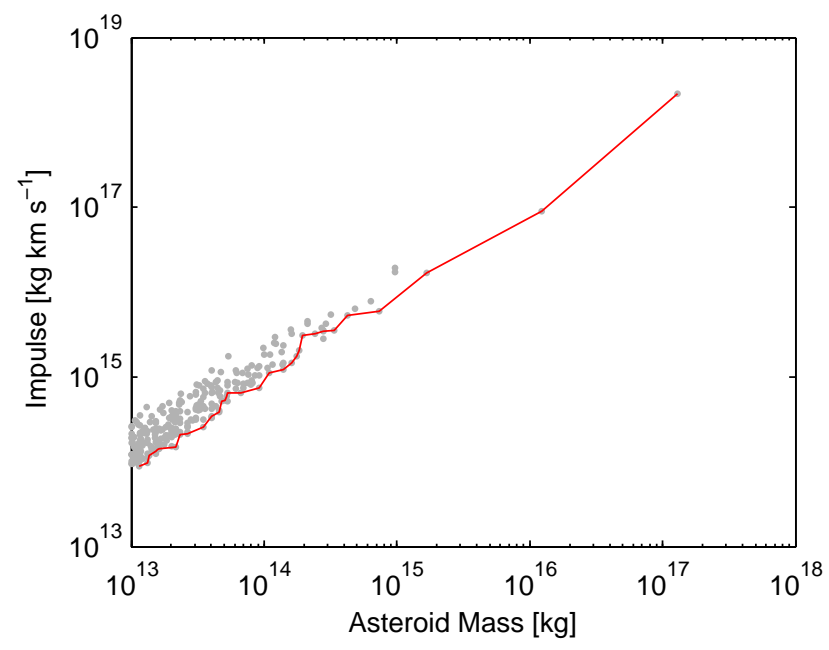

Figure 6: Impulse required for capture to the $\mathrm{L}_{1}$ point for the population of near Earth asteroids with masses above $1 \times 10^{13} \mathrm{~kg}$ with a Pareto front showing the optimum bodies for capture.

\section{Discussion}

Of importance to this method of geoengineering is knowledge about the lifetime of the dust grains within the zero velocity curve. There are several possibilities for natural loss of material from the dust cloud. These include collisions with the surface of the asteroid, variations in solar intensity, other solar effects such as the solar wind and coronal mass ejections, particle collisions leading to a spread of velocities and finally the escape of material smaller than the designed grain radius when assuming a certain size distribution.

It has been stated previously that the effect of solar radiation pressure is the dominant perturbation on the dust grains. Additionally, as the variation in the solar insolation over the course of the solar cycle is only $1-2 \mathrm{Wm}^{-2}$ [16] it is assumed that the effect that this has on the possible loss of material is negligible. The eccentricity of the Earth's orbit around the Sun will have a noticeable effect on the zero velocity curve around the asteroid. When placed at the conventional $\mathrm{L}_{1}$ position the volume at perigee and apogee varies by $\pm 5 \%$ with respect to the mean volume with a corresponding variation in the maximum insolation reduction of $\pm 0.1 \%$. This fluctuation in volume will not lead to any loss of material as the Jacobi constant of the bounding zero velocity curve will not change and hence the dust grains will remain bound.

Loss of material due to the size distribution of the dust grains is likely. The grain sizes below those desired will have a greater value for $\beta$ and thus the size of the zero velocity curve will decrease, thus decreasing the effectiveness of these smaller particles. Additionally, as the grains will be ejected with a similar velocity the smaller dust grains will have an energy above that of the bounding Jacobi constant and thus are likely to escape. For example, assuming a lognormal distribution with $\mu=-10.34$ and $\sigma=0.25,21 \%$ of the mass ejected has a radius below $32 \mu \mathrm{m}$ and hence is likely to escape. Conversely for a greater standard deviation of $\sigma=0.5$ only $6.5 \%$ will escape due to the mass being dominated by the larger particle sizes.

The motion of dust grains around an asteroid is a non-trivial problem as discussed in [13] where it is noted that the majority of particles randomly ejected, in a simulation of a meteorite impact, will fall into one of two short lived classes of orbit; immediate re-impact or immediate escape. As the escape velocity is known then this secopnd catagory can be avoided and therefore avoiding immediate re-impact is the most important issue. The large number of random velocities that fall into the immediate re-impact category is due to the origin of the orbit being on the surface of the asteroid. Other classes of orbit exist and are expected to be more long lived, up to the region of hundreds of days, and are acheived through 


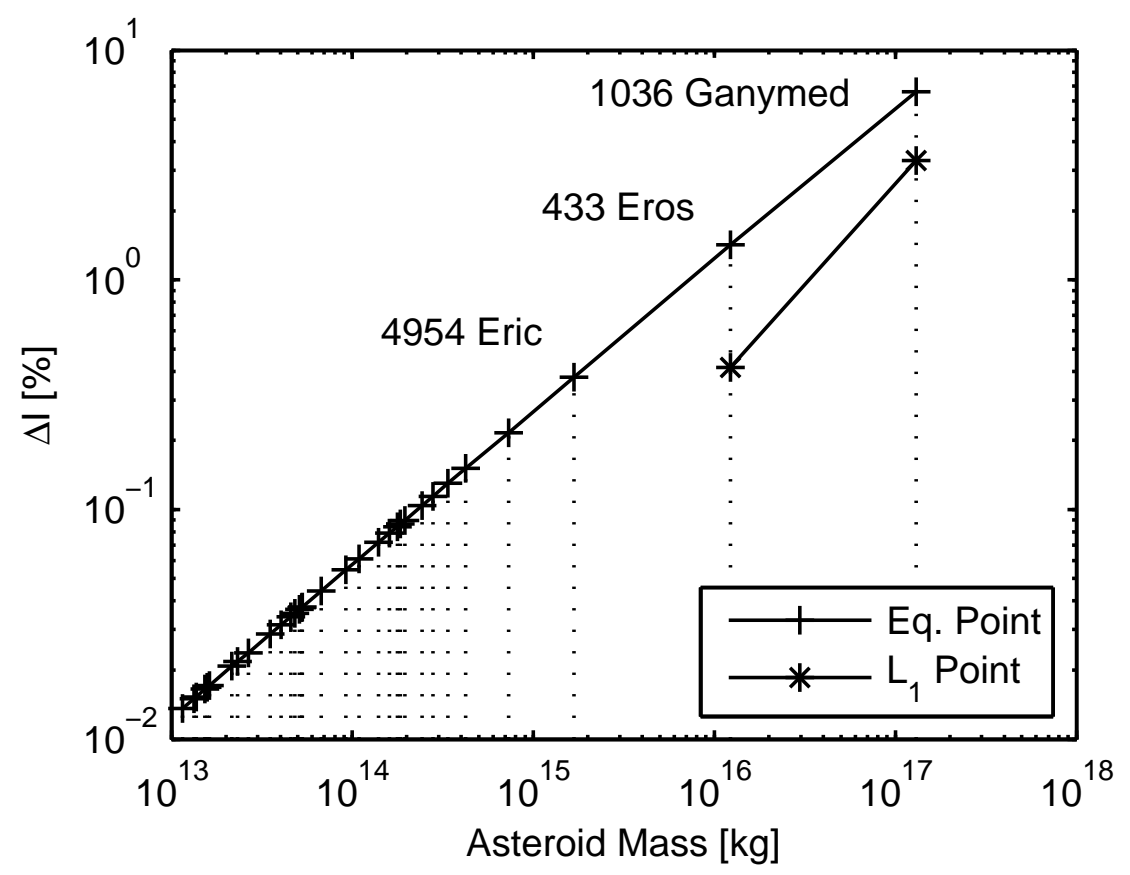

Figure 7: Maximum insolation change available for the masses of the asteroids on the Pareto front situated at the displaced equilibrium position and the classical $\mathrm{L}_{1}$ point with labels for the three largest bodies.

the specific selection of the initial conditions [12]. It is assumed here that the grains can be given the correct ejecta velocity to achieve long lived orbits whilst it is conservatively assumed that the grains with a $\beta$ value greater than 0.005 will escape very quickly.

Previously it has been shown that the collision timescale is long and thus it can be assumed that the velocities of the grains will not follow a Maxwell-Boltzmann distribution meaning there will be little spread in the speed. Therefore it is assumed that this will have a negligible effect on particle escape. For the grains that escape due to higher values of $\beta$ the useful lifetime will be greater than their lifetime within the zero velocity curve as they will be in a position to block solar radiation for approximately 50 days after escape [2].

An approximate calculation can now be made of the rate that material must be ejected from the surface to achieve the required $1.7 \%$ reduction in insolation. The number density of particles of radius $32 \mu \mathrm{m}$ required is $120 \mathrm{~m}^{-3}$ giving, for a total volume of $1 \times 10^{19} \mathrm{~m}^{3}$, a cloud mass of $6 \times 10^{11} \mathrm{~kg}$. Now assuming a particle lifetime of 180 days before re-impact gives an ejection rate of $1.4 \times 10^{8} \mathrm{~kg} \mathrm{hr}^{-1}$. The reduced efficiency of the system due to the dust size distribution can be approximated. For a lognormal mean of $\mu=-10.34(32 \mu \mathrm{m})$ and standard deviations of $\sigma=0.25$ and $\sigma=0.5$ the mean masses are $1.4 m_{32 \mu m}$ and $3.2 m_{32 \mu m}$ respectively whilst the ratio of the mean cross sectional area of the distribution and that of a $32 \mu \mathrm{m}$ radius particle are 0.81 and 1.42 . This gives final ejection rates that are 1.7 and 2.2 times larger than the nominal case. These mass ejection rates are large, however, should the technology become available to capture such an asteroid become available these mass ejection rates may not be unfeasible.

The loss timescale suggested here is short in comparison to the probable lifetime necessary for geoengineering, which is likely to be for several decades or even centuries. This will reduce the risks associated with this method and enables some control by simply decreasing the ejection rate of material. Should the insolation reduction need to be immediately stopped due to major unforeseen side effects then the asteroid can be displaced away from the $\mathrm{L}_{1}$ point and allowed to drift away. It will still be prudent to test the safety and efficiency of the method suggested here by first capturing a small asteroid 5-10m in 
size to the $\mathrm{L}_{1}$ point. Then the station keeping and dust ejection mechanisms as well as the ability of the dust to block sunlight on a small scale can be tested as well as verifying that solar radiation pressure is the dominant perturbation on the dust grains. Subsequently a larger asteroid $100-200 \mathrm{~m}$ in size, can be captured to test the principles of the zero velocity curve and affect a small insolation reduction on the Earth to verify the method of geoengineering. Additionally, for a short period of time, the principles discussed in [2] can be used to generate a larger reduction in insolation to determine whether there are any unintended side effects with large scale solar radiation management. Subsequently the necessity for such large scale geoengineering schemes can be assessed before continuing as well as the cost efficiency of this method of space based geoengineering.

\section{Conclusion}

A novel method of space-based geoengineering has been presented which uses the mass of a captured near Earth asteroid to gravitationally anchor a cloud of unprocessed dust in the vicinity of the $\mathrm{L}_{1}$ position to reduce the level of solar insolation at Earth. The captured asteroid also provides the source of dust in-situ. It has subsequently been shown that a cloud contained within the zero-velocity curve of the largest near Earth asteroid, Ganymed, can lead to an insolation reduction of $6.58 \%$ which is significantly larger than the $1.7 \%$ required to offset the worst effects of a global warming of $2{ }^{\circ} \mathrm{C}$. The masses of the next largest near Earth asteroids are not quite large enough to achieve the required level of insolation reduction, however, they are significant enough to be used as part of a portfolio of geoengineering schemes.

\section{Acknowledgements}

The work reported in this paper was funded by the European Research Council through VISIONSPACE (project 227571).

\section{References}

\section{References}

[1] Angel, R., Feasibility of cooling the Earth with a cloud of small spacecraft near the inner Lagrange point (L1), Proceedings of the National Academy of Sciences, 103, 17184-17189, 2006.

[2] Bewick, R., Sanchez, J. P., McInnes, C. R., The feasibility of using an $\mathrm{L}_{1}$ positioned dust cloud as a method of space-based geoengineering, Advances in Space Research, 49, 1212-1228, 2012.

[3] Bowell, E., Hapke, B., Dominique, et al., Application of photometric models to asteroids, Asteroids II. University of Arizona Press, Tucson, pp. 524-556, 1989.

[4] Chesley, S. R., Chodas, P. W., Milani, A., and Yeomans, D. K. Quantifying the Risk Posed by Potential Earth Impacts, Icarus, 159, 423-432, 2002.

[5] Govindasamy, B. and Caldeira, K., Geoengineering Earth's radiation balance to mitigate CO2induced climate change. Geophysical Research Letters 27, 2141-2144, 2000

[6] IPCC, Contribution of Working Groups I, II and III to the Fourth Assessment Report of the Intergovernmental Panel on Climate Change, Core Writing Team, Pachauri, R.K. and Reisinger, A. (Eds.), IPCC, Geneva, Switzerland. pp 104, 2007.

[7] McInnes, C.R., Space-based geoengineering: challenges and requirements, Proceedings of the Institution of Mechanical Engineers, Part C: Journal of Mechanical Engineering Science, 224, 571-580, 2010 . 
[8] Olds, J., Charania, A., Graham, M., et al., The League of Extraordinary Machines: A Rapid and Scalable Approach to Planetary Defense Against Asteroid Impactors, NASA Institute for Advanced Concepts, 30th April, 2004.

[9] Pearson, J., Oldson, J. and Levin, E., Earth rings for planetary environment control, Acta Astronautica, 58, 44-57, 2006.

[10] Sanchez, J.P. and McInnes, C., Asteroid Resource Map for Near-Earth Space, Journal of Spacecraft and Rockets, 48, 153-165, 2011.

[11] Schaub, H. and Junkins, J.L., Analytical Mechanics of Space Systems, AIAA, 2003.

[12] Scheeres, D.J., Ostro, S.J., Hudson, R.S., et al., Dynamics of Orbits Close to Asteroid 4179 Toutatis, Icarus 132, 53-79, 1998

[13] Scheeres, D.J., Durda, D.D. and Geissler, P.E., The fate of asteroid ejecta, in: Bottke, W.F., Cellino, A., Paolicchi, P., et al. (eds) Asteroids III, University of Arizona Press, Tucson, p.527-544, 2002

[14] Struck, C., The feasibility of shading the greenhouse with dust clouds at the stable lunar Lagrange points, JBIS, 60, 82-89, 2007.

[15] Wilck, M. and Mann, I., Radiation pressure forces on "typical" interplanetary dust grains, Planetary and Space Science, 44, 493-499, 1996.

[16] Willson, R. C. and Hudson, H. S., The Sun's luminosity over a complete solar cycle, Nature, 351, $42-44,1991$ 